\title{
Research Themes and Hotspots in Nursing Informatics Education Based Co-Word Analysis
}

\author{
Jialin LIU ${ }^{\mathrm{a}, 1}$, Siru LIU ${ }^{\mathrm{b}}$, Yi YANG ${ }^{\mathrm{a}}$ and Jinbo FANG ${ }^{\mathrm{c}}$ \\ ${ }^{a}$ Department of Medical Informatics, West China Hospital, Sichuan University, China \\ ${ }^{\mathrm{b}}$ Department of Biomedical Informatics, University of Utah, Salt Lake City, Utah, USA \\ ${ }^{\mathrm{c}}$ West China School of Nursing, West China Hospital, Sichuan University, China
}

\begin{abstract}
The purpose of the study is to identify research topics and hotspots in nursing informatics education during the period of 2009-2018. The relevant literature of nursing informatics education was retrieved from the Web of Science Core Collection (WoSCC). This study identified three research themes and hotspots in nursing informatics education using co-word analysis. The themes are curriculum, technology, and nursing informatics. The results provide useful information for researchers to research topic choices and in-depth research.
\end{abstract}

Keywords. Nursing informatics, education, co-word analysis

\section{Introduction}

Nursing informatics is critical for nursing and the whole healthcare system [1]. Nursing informatics education is essential for nurses to provide safe, effective and high-quality care in the digital hospital environment. In order to better understanding the current status of nursing informatics education, we used the co-word analysis to study the themes and hotspots in nursing informatics education.

\section{Methods}

All of the literature was retrieved from the WoSCC (from January 1, 2009, to December 31,2018 ) by two reviewers independently, and used the topic terms "nursing informatics" and "education" to search. The co-word analysis included only articles, proceeding papers, reviews, and meeting abstracts in the English language. Co-word analysis is to abstract the keyword list in the research field to mark the literature and reflect its core contents [2]. VOSviewer v.1.6.8 (a computer program for constructing and visualizing maps) was used to create a knowledge map.

${ }^{1}$ Corresponding Author, Jialin Liu, Department of Medical Informatics, West China Hospital, Sichuan University, Chengdu, Sichuan, China; E-mail: dlj18@163.com. 


\section{Results}

A total of 346 articles were retrieved in WoSCC from 2009 to 2018. From the screening of the titles, abstracts and full texts, 168 unrelated articles, five other document type articles, and five non-English articles were excluded. In total, 168 articles were identified. There were 556 keywords extracted from the 168 articles. Among 556 keywords, 32 highly frequent keywords met with minimum co-occurrences of five. The 32 keywords were divided into three clusters (Table 1). The results show a grouping around three clusters (keywords visualization using VOSviewer). Figure 1 shows the network visualization map of the keywords. These clusters are represented by different colors. Figure 2 presents a timeline view of various topics. The color of a term indicates the term's average publication year (the label with yellow indicates more recent publications).

Table 1. Co-word clusters of keywords.

\begin{tabular}{lll}
\hline Cluster & Theme & \multicolumn{1}{c}{ keywords } \\
\hline 1 Red & Curriculum & Education, nursing, competence, informatics, knowledge, computer \\
& $(16)$ & literacy, skills, medical informatics, information technology, et al \\
2 Green & Technology & Care, attitudes, computers, electronic health records, health information \\
& $(8)$ & technology, quality. \\
\multirow{3}{*}{3 Blue } & Nursing informatics & Nursing education, internet, competencies, informatics competencies, \\
& $(8)$ & educational technology, nursing education research.
\end{tabular}

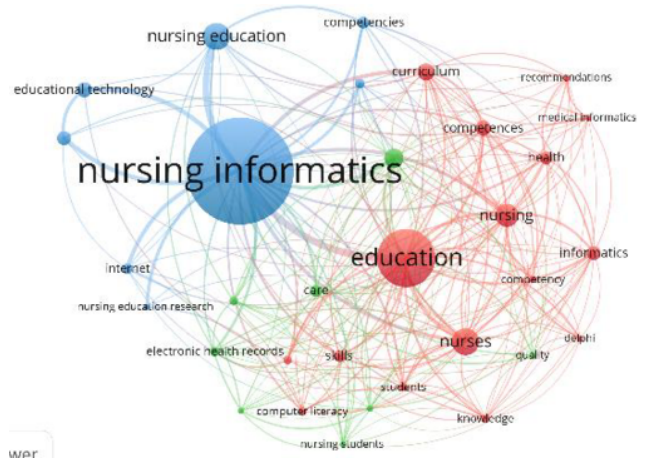

Figure 1. Network visualization of the keywords

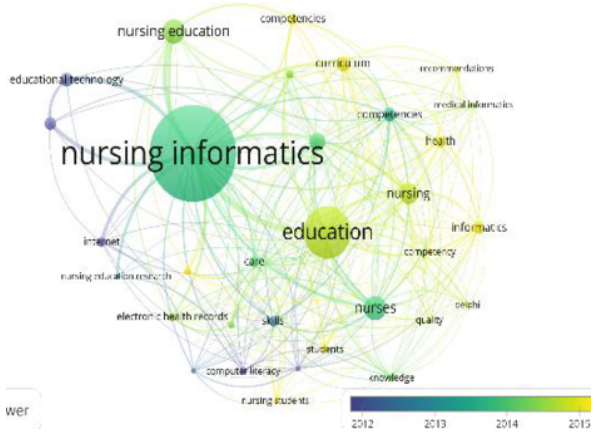

Figure 2. Overlay visualization of the keywords

\section{Conclusion}

Three clusters represent different research themes and directions on nursing informatics education. The co-word analysis will help in tracking the research themes and hotspots in nursing informatics education. 


\section{References}

[1] Cummins MR, Sward K, Guo JW. Leaders in nursing informatics education and research: The University of Utah celebrates 25 years. Comput Inform Nurs 33(2015),379-381.

[2] Borner K, Chen CM, Boyack KW. Visualizing knowledge domains. Annual Review of Information Science and Technology 37(2003),179e-255e. 